\title{
INDETERMINATEZZA METAFISICA E MECCANICA QUANTISTICA
}

\author{
CLAUDIO CALOSI (*)
}

\begin{abstract}
SunTO. - Nel lavoro si argomenta che la meccanica quantistica offre esempi di genuina indeterminatezza metafisica per quanto rigaurda l'istanziazione di alcune proprietà. Si argomenta inoltre che non si riesce a rendere conto di tale indeterminatezza quantistica in termini puramente modali. Il miglior modo di renderne conto, si suggerisce, è riconoscere che esistano sistemi fisici che istanziano una proprietà determinabile, ma non un'unica proprietà determinata di tale determinabile.
\end{abstract}

$* * *$

ABSTRACT. - The paper argues that quantum mechanics provide examples of genuine metaphysical indeterminacy that cannot be accounted for in purely modal terms. The best account of quantum indeterminacy has it that quantum systems have determinable properties without thereby having a unique determinate of that determinable.

\section{INTRODUZIONE}

L'indeterminatezza metafisica (IM) viene spesso caraterizzata come quel tipo di indeterminatezza che non è dovuta nè a deficienza semantica, nè a limitazione epistemica. IM sarebbe piuttosto un fenomeno genuinamente metafisico. Questo perchè la fonte dell'indeterminatezza in questione sarebbe il mondo stesso. In altre parole sarebbe il mondo stesso (sotto qualche rispetto) a essere indeterminato, non la nostra conoscenza del mondo, nè il linguaggio che usiamo per descriverlo.

Fenomeni molto diversi tra loro potrebbero dare vita a casi di IM.

(*) Università di Ginevra, Svizzera. E-mail: claudio.calosi@unige.ch 
Secondo alcuni ci sarebbero casi di IM tipicamente composizionale: sarebbe la struttura mereologica di una certa entità a essere genuinamente indeterminata o vaga. ${ }^{2}$ Altri hanno sostenuto che i futuri contingenti in particolari metafisiche del tempo - la cosiddetta moving spottight view - costituirebbero un altro caso. ${ }^{3}$ Infine, ci sarebbero casi tipicamente quantistici. In realtà si è sostenuto che la meccanica quantistica (MQ) offra due esempi distinti di IM: indeterminatezza dell'identità degli oggetti quantistici, ${ }^{4}$ e indeterminatezza delle proprietà quantistiche. In particolare quest'ultima sarebbe dovuta al fallimento del principio classico secondo cui ogni sistema fisico ha un valore determinato di ogni proprietà di cui gode. In questo lavoro non si discuteranno quali siano le relazioni tra questi due tipi di indeterminatezza quantistica. In effetti, il lavoro si concentra esclusivamente sull'ultima, i.e. sull'indeterminatezza delle proprietà quantistiche. In particolare, si vedrà come uno dei più influenti account metefisici di IM incontri delle difficoltà nel rendere conto di tale indeterminatezza. Si suggerirà allora un account alternativo.

\section{SUPERVALUTAZIONISMO METAFISICO}

Molto probabilmete, l'account di IM più influente è il cosidetto supervalutazionismo metafisico - per analogia al più noto supervalutazionismo semantico - di Barnes e Williams (2011). Ecco una prima caratterizzazione:

Supervalutazionismo metafisico (SM). IM è letteralmente indeterminatezza tra diversi stati di cose massimamente precisi. Si ha IM quando è indeterminato quale stato di cose completamente determinato si dia.

1 Si veda ad esempio Sattig (2014).

2 La letteratura metafisica spesso distingue tra vaghezza e indeterminatezza. Qui si useranno i due termini come sinonimi.

3 Si veda Cameron (2015).

4 Si veda French e Krause (2003).

5 Questo scritto è il risultato di un lavoro comune con Jessica Wilson (Università di Toronto). In effetti, l'account alternativo che si intende sviluppare nel contesto quantistico è suo. Eventuali errori o imprecisioni sono tuttavia soltanto miei. 
Si noti che SM, anche nella formulazione imprecisa di cui sopra, è un account primitivista e anti-riduzionista, nel senso che IM non è definita a partire da altre nozioni, nè riducibile a queste. L'idea è generale alla base di SM viene commentata da Darby (2010) come segue:

The approach I have in mind has it that indeterminacy may be captured by a modal operator. By this I mean that a 'definitely' operator $D$ may be prefixed to formulas; that the indeterminacy operator $\nabla$ is to $D$ as contingency is to necessity ( $\nabla \phi$ iff $\neg D \phi \wedge \neg D \neg \phi)$; that something like a possible-worlds semantics may be given; and that one may wonder what modal logic the D operator obeys (Darby, 2010: 234).

Dato un classico framework ersatzista (linguistico) di mondi possibili si può essere più precisi e definire: ${ }^{6}$

Possibilità precisificazionale. Un mondo ersatz $w_{1}$ è una possibilità precisificazionale $(\mathrm{PP})$ per un mondo $w_{2}$ sse $w_{1}$ non rappresenta in modo determinatamente scorretto $w_{2}$, i.e. $\forall p\left(p \in w_{1} \rightarrow \neg D \neg p_{w_{2}}\right){ }^{?}$

Precisificazione attuale. Un mondo $w$ è una precisificazione attuale (PA)del mondo attuale@sse w=PP(@).

Indeterminatezza metafisica (IM). Un mondo $w$ è IM sse esiste un insieme di mondi distinti $W=\left(w_{1}, \ldots, \mathrm{w}_{n}\right)$ tali che, per ciascuno dei $\mathrm{w}_{i} \in W$, si ha $\mathrm{w}_{i}=P P(@)$.

\section{INDETERMINATEZZA QUANTISTICA}

Come si è detto nell'introduzione l'indeterminatezza quantistica deriva dal fatto che molte proprietà - o, in gergo quantistico, osservabili - non hanno un valore definito. Per euristica, si consideri la cosiddetta "interpretazione ortodossa" di MQ, insieme con il seguente principio:

6 La ricostruzione segue Mariani e Torrengo (MS).

7 Usando quantificazione a ordini superiori. Quando un mondo $w$ compare come pedice di una proposizione $p, w$ rappresenta il mondo a cui $p$ viene valutata. 
Legame autostato-autovalore (LAA). Un sistema fisico $s$ ha un valore determinato $v$ di una osservabile $O$ sse lo stato di $s$ è un autostato di $O$ corrispondente all'autovalore $v$.

Dato LAA non è difficile notare come la MQ offra tre diverse fonti di IM, corrispondenti a sovrapposizione di stati, esistenza di osservabili incompatibili e entanglement. In ciascuno di questi casi è facile provare che esiste almeno un sistema fisico $s$ in un dato stato quantistico e una osservabile $O$ tale che lo stato di $s$ non è un autostato di $O$. Segue direttamente da LAA che $s$ non ha un valore determinato di $O$. Questa è la forma di IM tipicamente quantistica di cui si vuole rendere conto. ${ }^{8}$

\section{SuPERVAluTAZIONISMO QUANTISTICO}

Il supervalutazionismo metafisico è innegabilmente un account semplice ed elegante di IM. Non sorprende quindi che molti si siano chiesti come possa essere esteso per rendere conto dell'indeterminatezza quantistica. Come nota Darby, si potrebbe addirittura essere ottimisti in proposito:

[There is] a suggestive parallel between the terms in the superposition and the idea [...] of precisifications. One of the terms in the superposition [... ] is a term where the cat is alive, the other is not; that is reminiscent of multiple ways of drawing the extension of 'alive', on some of which 'the cat is alive' comes out true, on some, false. (Darby, 2010: 235)

Tuttavia MQ sembra offrire due importanti argomenti contro SM, che chiamerò Argomento dal Teorema Kochen-Specker e Argomento delle Osservabili Incompatibili rispettivamente.

\subsection{L'argomento dal Teorema Kochen-Specker}

Informalmente, il teorema di Kochen-Specker afferma che MQ è inconsistente con la congiunzione delle seguenti affermazioni:

8 Qui si assumerà che si diano casi di indeterminatezza quantistica. Per una posizione diversa si veda Glick (2017). 
Non-Contestualismo. Se un sistema quantistico $s$ istanzia ${ }^{9}$ una proprietà determinata $O=v, s$ istanzia $O=v$ indipendentemente dal contesto di misurazione di $O$.

Definitezza dei Valori. Ogni osservabile quantistica $O$ di un sistema $s$ ha un valore definito $O=v$.

In altre parole, ogni interpretazione non-contestualista di MQ è costretta a riconoscere che non è possibile avere valori determinati per tutte le osservabili quantistiche definibili per un sistems fisico $s$. L'argomento contro SM diventa allora semplice. SM richiede che ogni PA sia massimamente precisa, i.e. che il valore di ogni osservabile quantistica sia completamente determinato. Il teorema di Kochen-Specker afferma che non è possibile. ${ }^{10}$

Per comprendere meglio, si consideri il seguente esempio fisicamente inaccurato. ${ }^{11}$ Sia $s$ un sistema fisico in @ tale che $s$ è in un autostato di $\operatorname{spin}_{x}$ -up, i.e. $\left.|\psi\rangle_{s}=\mid \uparrow_{x}\right)_{s}$. Allora, secondo MS l'insieme $W$ di mondi che sono PA di @ contiene quattro elementi distinti, i.e. $W=\left(w_{1}, w_{2}, w_{3}, w_{4}\right)$, dove:

$$
\begin{aligned}
w_{1} & =\left\{\uparrow_{x}, \uparrow_{y}, \uparrow_{z}\right\} \\
w_{2} & =\left\{\uparrow_{x}, \uparrow_{y}, \downarrow_{z}\right\} \\
w_{3} & =\left\{\uparrow_{x}, \downarrow_{y}, \downarrow_{z}\right\} \\
w_{4} & =\left\{\uparrow_{x}, \downarrow_{y}, \uparrow_{z}\right\}
\end{aligned}
$$

Ciascuno dei $w_{i} \in W$ viola il teorema di Kocken-Specker. Si potrebbe avere una formulazione ulteriore dell'argomento appena discusso, più controversa, ma sicuramente istruttiva. Sia $p_{i}$ la proposizione che descrive la parte rilevante di $w_{i}$ di cui sopra. ${ }^{12}$ Ad esempio, sia $p_{1}=$ "il sistema $s$ ha spin- $x$ up, spin- $y$ up e spin- $z$ up". Allora $p_{1}$ è determinatamentefalsa a @, dato il teorema di Kochen-Specker. Dunque $w_{1}$

9 Uso consapevolmente il termine "istanziazione" per ragioni che saranno ovvie in $\S 6$.

10 Questo è in qualche modo l'argomento classico contro SM. Si veda ad esempio Darby (2010) e Skow (2010).

11 Tale inaccuretezza nasce dal fatto che il teorema di Kocken-Specker si applica a spazi di Hilbert con dimensione maggiore di 3 .

12 In particolare si ha che $p_{1} \in w_{1}$. 
rappresenta @ in modo determinatamente scorretto. Ne segue che $\neg\left(w_{1}\right.$ $=P P(@)$ ), contro SM. Il lettore attento si sarà accorto di quanto controverso sia questo modo di formulare l'argomento. Si potrebbe in effetti insistere che deriva dal teorema di Kocken-Specker che $p_{1}$ è falsa a@, ma non determinatamente falsa.

\subsection{L'argomento delle osservabili incompatibili}

L'esempio della sezione precedente aiuta anche a formulare un altro argomento quantistico contro SM. Si consideri un qualsiasi mondo $w_{i} \in W$. Non solo ogni $w_{i}$ è incompatibile con il teorema di KochenSpecker. Ogni $w_{i}$ costituisce anche una violazione del cosiddetto principio di indeterminazione generalizzato. Due qualsiasi osservabili incompatibili - o non commutabili, i.e. due osservabili $O_{1}, O_{2}$ tali che $\left[O_{1}, O_{2}\right]=O_{1} O_{2}$ - $\mathrm{O}_{2} \mathrm{O}_{1} \neq 0$ - obbediscono a tale principio. Questo implica che una massima determinatezza nel valore di una osservabile $O_{1}$ implica una massima indeterminatezza nel valore dell'osservabile non commutabile $\mathrm{O}_{2}$. Esempi classici di osservabili non commutabili includono la posizione e il momento, e le componenti di spin lungo le tre direzioni spaziali. L'argomento dall'esistenza di osservabili incompatibili contro SM è ancora una volta molto semplice. SM richiede che ogni PA sia massimamente precisa. MQ implica che, qualora una precisificazione PA coinvolga osservabili incompatibili, questo non è possibile. L'argomento in questione sembra essere addirittura più forte dell'argomento dal teorema di Kocken-Specker perchè l'esistenza di osservabili incompatibili è una componente quasi inevitabile di (qualsiasi interpretazione di) MQ.

Per avere una controparte della formulazione più controversa dell'argomento dal teorema di Kochen-Specker, sia $p$ la seguente proposizione: "il sistema fisico $s$ ha un preciso valore $q_{x}$ della posizione e un preciso valore del momento $p_{x}$ ". Allora, dato il caso particolare in questione, deriva dal principio di indeterminatezza di Heisenberg, che $p$ è determinatamente falsa a @. Dunque un mondo $w$ tale che $p \in w$ rappresenta@ in modo determinatamente scorretto. Ne segue che $\neg(w=$ PP(@)), contro SM.

\section{L'ACCOUNT DETERMINABILE}

Recentemente, Jessica Wilson ha sviluppato un account comple- 
tamente diverso di IM, basato sulla distinzione tra proprietà determinabili e determinate..$^{13} \mathrm{~A}$ una prima approssimazione,

Determinables and determinates are in the first instance type-level properties that stand in a distinctive specification relation: the "determinable determinate" relation (for short, "determination"). For example, color is a determinable having red, blue, and other specific shades of color as determinates; shape is a determinable having rectangular, oval, and other specific (including many irregular) shapes as determinates; mass is a determinable having specific mass values as determinates (Wilson, 2017: 1).

La relazione di determinazione è solitamente caratterizzata da diversi assiomi che si suppone ne governino il comportamento. Per quanto segue, due sono i principi più importanti: ${ }^{14}$

Determinazione. Per ogni determinabile massimamente non specifico $D$ esiste un determinato massimamente specifico $d$ tale che, ogni sistema $s$ che istanzia $D$ istanzia $d$.

Unicità della Determinazione. Per ogni determinabile massimamente non specifico $D$ esiste un unico determinato massimamente specifico $d$ tale che, ogni sistema $s$ che istanzia $D$ istanzia $d$.

Secondo l'account determinabile, IM consiste nel darsi di uno stato di cose in cui almeno uno degli oggetti costituenti istanzia un determinabile $D$ senza tuttavia istanziare un unico determinato $d$ di $D$. Nelle parole di Jessica Wilson:

What it is for a state of affairs to be MI in a given respect $R$ at a time $t$ is for the state of affairs to constitutively involve an object (more generally, entity) $O$ such that (i) $O$ has a determinable property $P$ at $t$, and (ii) for some level $L$ of determination of $P, O$ does not have a unique level- $L$ determinate of $P$ at $t$ (Wilson, 2013: 366).

Naturalmente ci sono due modi per un sistema fisico $s$ di istanzia-

13 Si veda Wilson (2013).

14 In quanto segue si restringe l'attenzione ai determinabili massimamente nonspecifici, e i determinati massimamente specifici. 
re $D$ senza istanziare un unico $d$ di $D$ : (i) $s$ non istanzia nessun $d$ di $D$, o (ii) $s$ istanzia più di un $d$ di $D$. In altre parole, nel primo caso, si viola Determinazione, mentre nel secondo caso si viola Unicità della Determinazione. Jessica Wilson chiama casi gappy di IM i primi casi, e casi glutty di IM i secondi.

\section{APPLICAZIONE QUANTISTICA DELL'ACCOUNT DETERMINABILE}

Nel resto del lavoro, invece di difendere la plausibilità generale dell'account determinabile, vorrei esplorare una particolare applicazione che non ha ricevuto quasi alcuna attenzione in letteratura e che trovo invece promettente. Prima di entrare nei dettagli, è meglio essere chiari sulle esplicite assunzioni che vengono fatte. In particolare, assumerò che le osservabili quantistiche hanno una struttura determinabile-determinato. In effetti, assumerò che i determinabili massimamente non-specifici siano matematicamente rappresentati da operatori lineari hermitiani, e che i determinati massimamente specifici siano rappresentati dagli autovalori possibili di tali operatori.

La particolare applicazione che si vuole discutere è una applicazione glutty, il cui tenet centrale - e più controverso - è che la relazione di istanziazione che sussiste tra una osservabile quantistica $O$ e un sistema $s$ possa darsi per gradi. Il miglior modo per comprendere la proposta è vederla al lavoro - per così dire - nei casi paradigmatici di indeterminatezza quantistica, i.e. sovrapposizione, osservabili incompatibili e entanglement.

\subsection{Sovrapposizione di stati}

Il suggerimento generale che si intende sviluppare è che il grado di istanziazione di una osservabile $O=v$ da parte di un sistema $S$ in uno stato quantistico $|\psi\rangle$ è dato dal quadrato del coefficiente dell'autostato di $O$ appartenente a $v$. Di conseguenza, si dovrebbe rimpiazzare LAA con una sua variante che tenga conto dei gradi di instanziazione, ad esempio:

Legame Autostato Autovalore per Gradi (LAAG). Un sistema fisico $s$ ha un valore determinato $v$ di una osservabile $O$ al grado $g$ sse $\sqrt{g}$ è il coefficiente dell'autostato di $O$ appartenente all'autovalore $v$. 
Più in dettaglio, si consideri un sistema $s$ in uno stato arbitrario $|\psi\rangle$, e si consideri una osservabile $O$ con due soli auostati $\left|\omega_{1}\right\rangle$ e $\left|\omega_{2}\right\rangle$ appartenti a due autovalori diversi, 1 e -1 rispettivamente. Per prima cosa, si scriva lo stato di $s$ usando gli autostati di $O$ come base. Il risultato è in generale la sovrapposizione: $|\psi\rangle=c_{1}\left|\omega_{1}\right\rangle+c_{2}\left|\omega_{2}\right\rangle$. Dato LAAG il sistema $s$ esemplifica IM in quanto istanzia $O=1$ e $O=-1$ a due gradi diversi. In particolare, (i) $s$ istanzia $O=1$ al grado $\left|c_{1}^{2}\right|$, e (ii) istanzia $O=-1$ al grado $\left|c_{2}^{2}\right|$. Non è difficile notare che questa è una variante dei casi glutty di IM.

\subsection{Osservabili incompatibili}

Il caso della sovrapposizione rappresenta il caso esemplare della proposta che si sta considerando. In effetti, ogni caso ulteriore viene prima ricondotto a una sovrapposizione, e poi viene seguito il procedimento appena descritto. Nel caso di osservabili incompatibili arbitrarie $O_{1}$ e $O_{2}$ la riconduzione a un caso di sovrapposizione è piuttosto diretta. Ogni autostato di $O_{1}$ è infatti una sovrapposizione di autostati di $O_{2}$ e vice-versa. Non si deve dunque che scrivere lo stato del sistema $s$ usando come base gli autostati dell'osservabile incompatibile, e poi procedere come in $\S 6.1$.

\subsection{Entanglement}

In un caso paradigmatico di entanglement si avrà un sistema quantistico $s_{12}$ composto da due sistemi distinti $s_{1}$ e $s_{2}$. Si ha IM quando $s_{12}$ è in un autostato di, e.g. $O_{12}=O_{1}-O_{2}$ definito sullo spazio tensore degli spazi di Hilbert per $s_{1}$ e $s_{2}$, ma $s_{1}$ e $s_{2}$ non sono in un autostato di $\mathrm{O}_{1}$ e $\mathrm{O}_{2}$ rispettivamente. La procedura per ricondurre tale caso a una semplice sovrapposizione è leggermente più complessa. Prima si calcolano gli stati ridotti di $s_{1}$ e $s_{2}$ a partire dallo stato di $s_{12}$, facendone la traccia. Gli stati ridotti così ottenuti sono tuttavia stati misti. Occorre dunque sfruttare il teorema secondo cui ogni stato misto è rappresentabile come una somma pesata di stati puri. Una volta arrivati a questa forma, si può procedere come in $\S 6.1$.

\section{Conclusioni}

Per riassumere: si è visto quali fenomeni tipicamente quantistici possano essere visti come fonte di indeterminatezza genuinamente 
metafisica. Si è osservato che il miglior account metafisico di tale indeterminatezza non è completamente soddisfacente e se ne è suggerito un altro, il cui tenet centrale è metafisicamente controverso. In effetti, le sfide aperte per l'account che ha occupato l'intera sezione $\$ 6$ sono, tra le altre, principalmente due: (i) come rendere conto metafisicamente dei gradi di istanziazione, e (ii) quale relazione ci sia tra gradi di istanziazione e probabilità oggettive, dato che entrambe sono date dalla regola di Born. Insomma, c'è ancora molto lavoro da fare all'incrocio tra (filosofia della) fisica e metafisica. E allora, meglio chiudere con queste parole del grande Tom Stoppard: $:^{15}$

Yelizaveta, when things get very small they get truly crazy (...) A hydrogen atom has a single electron flitting about like a moth in an empty cathedral (...) Every atom is a cathedral (...) There is a straight ladder from the atom to the grain of sand, and the only real mystery in physics is the missing rung. Below it, particle physics; above it, classical physics; but in between, metaphysics!

\section{RINGRAZIAMENTI}

La lista di persone che vorrei ringraziare sarebbe troppo lunga se mi dovessi soffermare su ogni nome. Tuttavia, uno almeno, di nomi, deve essere fatto. Grazie a Jessica Wilson che è il vero motore immobile di questo lavoro e di tanti altri.

15 Vedi Tom Stoppard, Hapgood. 


\section{BIBLIOGRAFIA}

Barnes E., Williams R. 2011. A Theory of Metaphysical Indeterminacy. In Bennett K., Zimmerman D. (Eds.) Oxford Studies in Metaphysics, Vol. 6, Oxford, Oxford University Press: 103-148.

Cameron R. 2015. The Moving Spotlight. Oxford: Oxford University Press.

Darby G. 2010. Quantum Mechanics and Metaphysical Indeterminacy. Australasian Journal of Philosophy, 88: 227-245.

French S. e Krause D. 2003. Quantum Vagueness. Erkenntnis, 59 (1): 97-124. Glick, D. 2017. Against Quantum Indeterminacy, Thought, DOI: 10.1002/tht3.250.

Sattig T. 2014. Mereological Indeterminacy: Metaphysical but Not Fundamental. In K. Akiba e A. Abasnezhad (eds.),Vague Objects and Vague Identity. New Essays on Ontic Vagueness. Berlin: Springer, pp. 2542.

Skow B. 2010. Deep Metaphysical Indeterminacy Philosophical Quarterly, 60: 851-858.

Wilson J. 2013. A Determinable-based Account of Metaphysical Indeterminacy. Inquiry, 56: 359-385.

Wilson J. 2017. Determinable and Determinates. Stanford Encyclopedia of Philosophy at: https://plato.stanford.edu/entries/determinate-determinables/ 